\title{
Effect of Comprehensive Rehabilitation Intervention on the Occurrence of Ventilator-Associated Pneumonia in Critical Patients
}

\author{
Z. LIU AND W. ZHANG*
}

Critical Respiratory Medicine, Affiliated Hospital of Inner Mongolia Medical University, Hohhot 010030, ${ }^{1}$ Central Sterile Supply Department, Affiliated Hospital of Inner Mongolia Medical University, Hohhot 010030, China

Liu et al.: Impact of comprehensive rehabilitation intervention on the occurrence of ventilator-associated pneumonia in critical patients

\begin{abstract}
In order to study the effect of comprehensive rehabilitation intervention on the occurrence of ventilatorassociated pneumonia in critical patients, this study selected the critical patients admitted to Affiliated Hospital of Inner Mongolia Medical University as the research object, and performed routine rehabilitation treatment (control group) and comprehensive rehabilitation treatment (observation group), recorded and compared the success rate of ventilator weaning, incidence of ventilator-associated pneumonia, mechanical ventilation time, length of hospital stays in intensive care unit, clinical pulmonary infection score and acute physiology and chronic health evaluation II score. The result shows that the success rate of the observation group was significantly higher than that of the control group. The difference was statistically significant. The incidence of ventilator-associated pneumonia, mechanical ventilation time and hospitalization time in the intensive care unit were significantly lower in the observation group than in the control group. The difference was statistically significant. Compared with prior treatment, the clinical pulmonary infection score and acute physiology and chronic health evaluation II score of the two groups were significantly lower after treatment, and the difference was statistically significant. The clinical pulmonary infection score of the observation group after treatment was significantly lower than that of the control group and the difference was statistically significant, while the acute physiology and chronic health evaluation II score of the observation group after treatment was lower than that of the control group, but the difference was not statistically significant. Therefore, through the study of the impact of comprehensive rehabilitation intervention on the occurrence of ventilator-associated pneumonia in critical patients, comprehensive rehabilitation treatment for critical patients is safe and effective and can be used as a routine treatment in clinical intensive care unit. Although there are still some shortcomings in the research process, it still provides some basis and ideas for more precise research.
\end{abstract}

Key words: Comprehensive rehabilitation therapy, critical patients, ventilator-associated pneumonia, mechanical ventilation

Ventilator-associated pneumonia (VAP) refers to the pulmonary parenchymal inflammatory response that occurs after $48 \mathrm{~h}$ of mechanical ventilation (MV) treatment to $48 \mathrm{~h}$ after extubation ${ }^{[1,2]}$. Pneumonia occurs within $4 \mathrm{~d}$ after $\mathrm{MV}$ is called early-onset VAP, and pneumonia occurs more than $5 \mathrm{~d}$ later is called late-onset $\mathrm{VAP}^{[3]}$. The incidence of VAP is related to infection, and the pathogen is usually Gram-negative bacteria $^{[4]}$. The clinical symptoms of the disease are usually fever higher than $38^{\circ}$, the respiratory tract produces a lot of purulent secretions and the lungs have wet rale ${ }^{[5]}$. VAP is an important type of hospital acquired pneumonia (HAP) and is one of the most common and serious complications in $\mathrm{MV}^{[6]}$. Once the disease occurs, it is likely to cause difficulties on ventilator weaning, prolong the time of hospital of patients, and even threaten the patient's life safety, leading to death ${ }^{[7]}$.

Rehabilitation is a special treatment for preventing, improving and restoring the dysfunction and hypofunction of the patient's body through passive exercise therapy, anti-respiratory exercise therapy, active exercise therapy, etc ${ }^{[8]}$. Among them, the

*Address for correspondence

E-mail: zhangwei89974@163.com

Special Issue 8, 2020 
rehabilitation of the lungs is to perform targeted exercise, breathing training, publicity education and psychological support through comprehensive evaluation of the patients ${ }^{[9]}$. Rehabilitation of the lung can provide a good comprehensive respiratory therapy for patients with lung disease, improve their cardiovascular endurance, spontaneous breathing and self-care ability ${ }^{[10]}$. In addition, lung rehabilitation can also correct hypoxemia, reduce pulmonary artery pressure, delay pulmonary heart disease, prolong survival time and improve quality of life ${ }^{[1]}$.

In summary, although comprehensive rehabilitation therapy can reduce the incidence of VAP in critical patients, the current treatment options are not clear. Therefore, this study explore the impact of comprehensive rehabilitation intervention on the occurrence of VAP in critical patients by selecting critical patients as a research object, and try to provide a reference for clinical prevention and treatment of VAP.

\section{MATERIALS AND METHODS}

\section{Patients:}

100 critical patients admitted to Affiliated Hospital of Inner Mongolia Medical University (between August 2015 and July 2018), aged 30-70 y and with an average age of $47.96 \mathrm{y}$ were selected. They were randomly divided into two groups: control group (50 cases) and observation group (50 cases). There were 26 males and 24 females in the control group, aged 32-70 y, with an average age of $48.07 \mathrm{y}$; 24 males and 26 females in control group, aged 30-70 y, with an average age of $47.85 \mathrm{y}$. There were no statistical differences between the two groups in terms of age, gender, occupation, and time of onset ( $p>0.05$ ), so the study was comparable. The informed consent signed by the patients or their family members was obtained and this study was approved by the medical ethics committee of Affiliated Hospital of Inner Mongolia Medical University.

Inclusion criteria: All patients met the criteria for MV therapy; patients with stable respiratory parameters and hemodynamics; patients with complete clinical data; patients with good clinical compliance; signed relevant informed consent.

Exclusion criteria: Patients with pulmonary infection before MV treatment; patients with MV time less than 48 $h$; patients with intracranial pressure greater than $20 \mathrm{~cm}$ water column; severely incomplete organ function such as cardiopulmonary; patients with immunodeficiency disease; patients in pregnancy or lactation.

\section{Intervention of different rehabilitation methods for critical patients:}

The patients in the control group were treated with routine rehabilitation. The method was as follows: nursing inspection 3 times a day, closely monitor the vital signs of the patients, and limit the visits of the family members. According to the patient's condition, symptomatic treatment may be proceeded, such as relieve cough resolve phlegm, medication, transfusion therapy and diet care, etc.

Comprehensive rehabilitation treatment was performed on the patients in the observation group. The intervention method was as follows: In addition to routine rehabilitation treatment for patients, the ventilator should be thoroughly disinfected every day. The disposable ventilator tube should be used for the patient and replaced in time; the usage time should not exceed $24 \mathrm{~h}$. In the case of the patient's condition, the bed is raised by $30^{\circ}-45^{\circ}$ according to the patient's comfort, the head position is changed regularly, and the soft pillow is placed at the sole of the foot. Wipe and rinse the patient's mouth every $6 \mathrm{~h}$ with a suitable oral cleansing agent according to the patient's oral condition. Artificial airway humidification is performed every $1 \sim 2 \mathrm{~h}$. The expectorant medicines or antibiotics can be used according to doctor's advice. If there is sputum scab in the respiratory tract, increase the dose of humidification medicine or increase the frequency of humidification. The patient's position is changed every $2 \mathrm{~h}$, turning over and knocking back to help the patient to perform active or passive joint movement. A transcutaneous electrical nerve stimulator (XY-KSJD-A, Anyang Xiangyu Medical Equipment Co., Ltd., China) is used to perform neuromuscular electrical stimulation on patients, including triceps, quadriceps, and dorsal carpal extensor muscle group and tibialis anterior muscle group. Put the patient in the supine position and place a suitable sandbag on the belly area to exercise his respiratory muscles. When a patient is found to have a sputum snoring sound in the lungs during auscultation, a closed sucking treatment should be performed in time. For patients who are intolerant of tracheal intubation, sedative medication should be given as needed and daily awakening is required. In addition, medical staff should patiently explain the knowledge of VAP to patients and improve their own attention.

\section{Ventilator weaning method:}

After the patient's condition improves, and his or 
her oxygenation is good, hemodynamics is stable, and recover the ability to spontaneously breathe, the ventilator weaning test can be considered. When the medical staff tests the patient's spontaneous breathing ability, if the shallow breathing index is lower than 105 , the respiratory rate is too fast or too slow, the heart rate is greater than 140 beats/min, the spontaneous respiratory tidal volume is greater than $4 \mathrm{ml} / \mathrm{kg}$, or the arterial oxygen saturation is lower than $90 \%$, MV should be continue. If the patient can maintain spontaneous breathing for more than $48 \mathrm{~h}$, the weaning is considered successful. Calculate and compare the success rate of the two groups of patients. The time required for the patient to start tracheal intubation to complete ventilator weaning and the length of hospital stays in the intensive care unit (ICU) were recorded and compared.

\section{Diagnostic criteria for VAP:}

According to the guidelines for the diagnosis and treatment of HAP (draft) developed by the respiratory diseases branch of Chinese Medical Association, the diagnostic criteria for VAP are as follows: under the premise of excluding pulmonary diseases such as tuberculosis, lung cancer, and atelectasis, the patient's chest radiography shows the infiltrating shadow in the lung manifests new inflammatory lesion compared with pre-ventilation; the lungs showed signs of consolidation, wet rales during auscultation, accompanied by fever, a large amount of purulent secretions in the respiratory tract, and new pathogens in bronchial secretions, or blood cells greater than $10.0 \times 10^{9} / \mathrm{L}$ and other diseases; the incidence is $48 \mathrm{~h}$ after using the ventilator. The incidence of VAP was analyzed and compared between the two groups.

\section{Evaluation criteria for Clinical Pulmonary Infection Score (CPIS) and Acute Physiology and Chronic Health Evaluation (APACHE) II Scores:}

The CPIS is a scoring system that combines the clinical, imaging, physiologic, and microbiological criteria for the severity of clinical lung infections, which can be used as a diagnostic criterion for VAP, in order to reduce unnecessary exposure to antibiotics. There were 7 items in the evaluation criteria, including body temperature, white blood cell count, tracheal secretion, oxygenation, chest X-ray, progression of lung infiltrates, and tracheal aspiration culture. The highest score was 12 points. The low CPIS indicates that the patient's condition is relieved. If the CPIS is higher than 6 points, it suggests that the patient is at high risk of death. The Higher the score, the more serious is the patient's condition.

The APACHE II is a scoring system for assessing the severity of a patient's condition, consisting of an acute physiology score, an age score, and a chronic health score. The acute physiology score included 12 physiological indicators with a total of 60 points; the age score was based on the following criteria: less than 45 y old ( 0 points), $45-55$ y old ( 2 points), $55-65$ y old ( 3 points), $65-75$ y old ( 5 points), greater than 75 y old (6 points); in the chronic health score, the emergency operation or non-surgical ICU is counted as 5 points, be hospitalized in ICU after selective operation is counted as 2 points, no diagnosis of chronic organ dysfunction or immune function inhibition is counted as 0 points. The highest score is 71 points, the higher the score, the more serious the patient's condition.

\section{Statistical method:}

Data processing and analysis were performed using Statistical Package for the Social Sciences (SPSS) 21.0 statistical software. Quantitative data consistent with normal distribution were described by mean \pm standard deviation. Comparison between the two groups was performed by two independent samples t-test or rank sum test. The comparison of different group rates was performed by chi-square test.

\section{RESULTS AND DISCUSSION}

The result of comparison is shown in Table 1, fig. 1a, the success rate of the control group was $60.0 \%$, and the success rate of the observation group was $92.0 \%$. It can be seen that the success rate of the observation group was significantly higher than that of the control group, and the difference was statistically significant $(* \mathrm{p}<0.05)$.

The result is shown in Table 2, fig. 1b. The incidence of VAP in the control group was $34.0 \%$, and the incidence of VAP in the observation group was $8.0 \%$. It can be seen that the incidence of VAP in the observation group was significantly lower than that in the control group, and the difference was statistically significant $\left({ }^{*} \mathrm{p}<0.05\right)$.

The result is shown in Table 3, fig. 1c, the average MV time of the control group was $13.54 \pm 4.75 \mathrm{~d}$, and

TABLE 1: COMPARISON OF THE SUCCESS RATE
\begin{tabular}{lc} 
OF WEANING BETWEEN THE TWO GROUPS \\
\hline Group & Success Rate of Weaning (\%) \\
\hline Observation group & 60.0 \\
Control group & $92.0^{*}$ \\
\hline
\end{tabular}

TABLE 1: COMPARISON OF THE SUCCESS RATE OF WEANING BETWEEN THE TWO GROUPS 


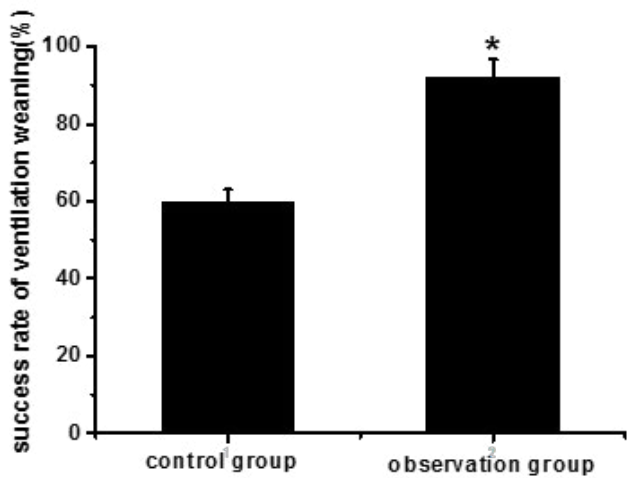

A

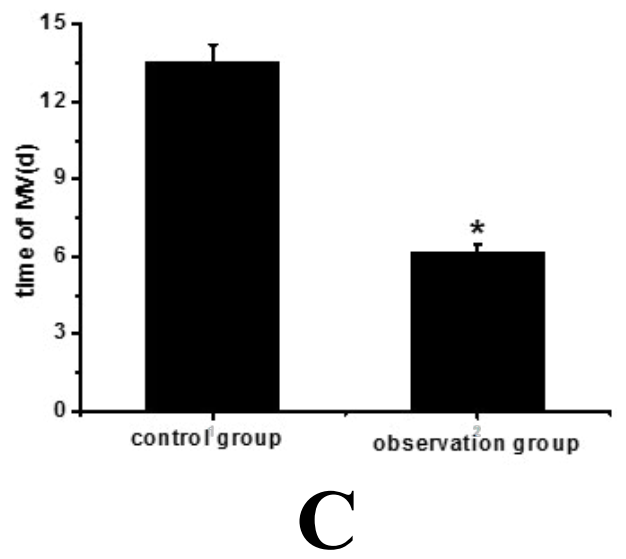

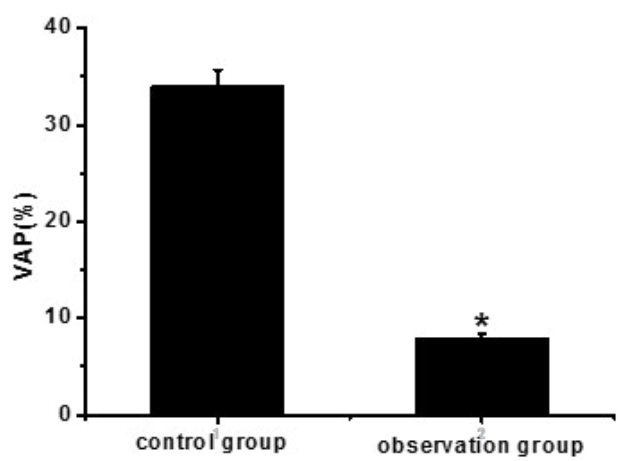

B

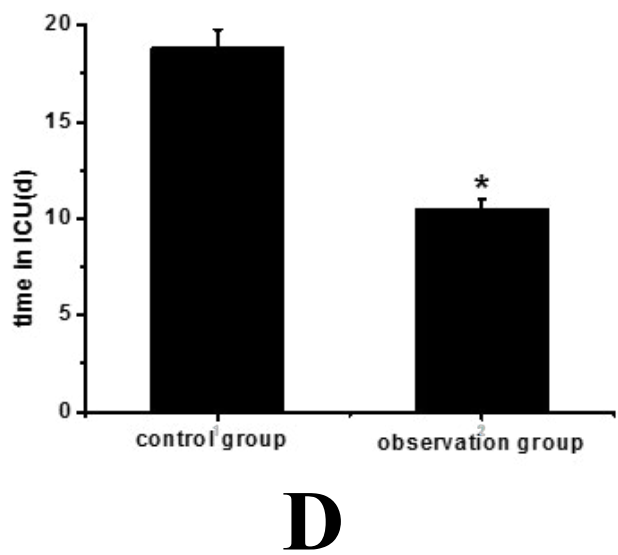

Fig. 1: Comparison in two groups

A. Comparison of the success rate of weaning between the two groups; B. Comparison of incidence of VAP in two groups; C. Comparison of MV time between the two groups; D. Comparison of hospital stays in ICU between two groups

TABLE 2: COMPARISON OF INCIDENCE OF VAP IN TWO GROUPS

\begin{tabular}{lc}
\hline Group & Incidence Rate of VAP (\%) \\
\hline Observation group & 34.0 \\
Control group & $8.0^{*}$ \\
\hline
\end{tabular}

TABLE 3: COMPARISON OF MV TIME BETWEEN THE TWO GROUPS

\begin{tabular}{lc}
\hline Group & MV Time \\
\hline Observation group & $13.54 \pm 4.75$ \\
Control group & $6.14 \pm 2.07^{\star}$ \\
\hline
\end{tabular}

the average MV time of the observation group was $6.14 \pm 2.07 \mathrm{~d}$. It can be seen that the MV time of the observation group was significantly lower than that of the control group, and the difference was statistically significant $(* \mathrm{p}<0.05)$.

The result is shown in Table 4, fig. 1d. The average ICU stay time in the control group was $18.84 \pm 5.37 \mathrm{~d}$, while the average ICU stay time in the observation group was $10.47 \pm 2.55 \mathrm{~d}$. It can be seen that the ICU stay time in the observation group was significantly lower than the control group, and the difference was statistically significant $(* \mathrm{p}<0.05)$.
TABLE 4: COMPARISON OF HOSPITAL STAYS IN ICU BETWEEN TWO GROUPS

\begin{tabular}{lc}
\hline Group & ICU Stay Time \\
\hline Observation group & $18.84 \pm 5.37$ \\
Control group & $10.47 \pm 2.55^{\star}$ \\
\hline
\end{tabular}

The result is shown in Table 5 and fig. 2. Compared with before treatment, the CPIS of the two groups were significantly lower after treatment $(\mathrm{p}<0.05)$, and the observation group was significantly lower than the control group, the difference was statistically significant $(p<0.05)$. Compared with before treatment, the APACHE II scores of the two groups were significantly lower after treatment $(p<0.05)$. The APACHE II score of the observation group after treatment was lower than that of the control group, but the difference was not statistically significant ( $\mathrm{p}>0.05)$.

In this study, the effects of comprehensive rehabilitation intervention on the occurrence of VAP in critical patients were studied. The critical patients admitted to Affiliated Hospital of Inner Mongolia Medical University were selected as the research subjects, and they were treated with conventional rehabilitation (control group) and 

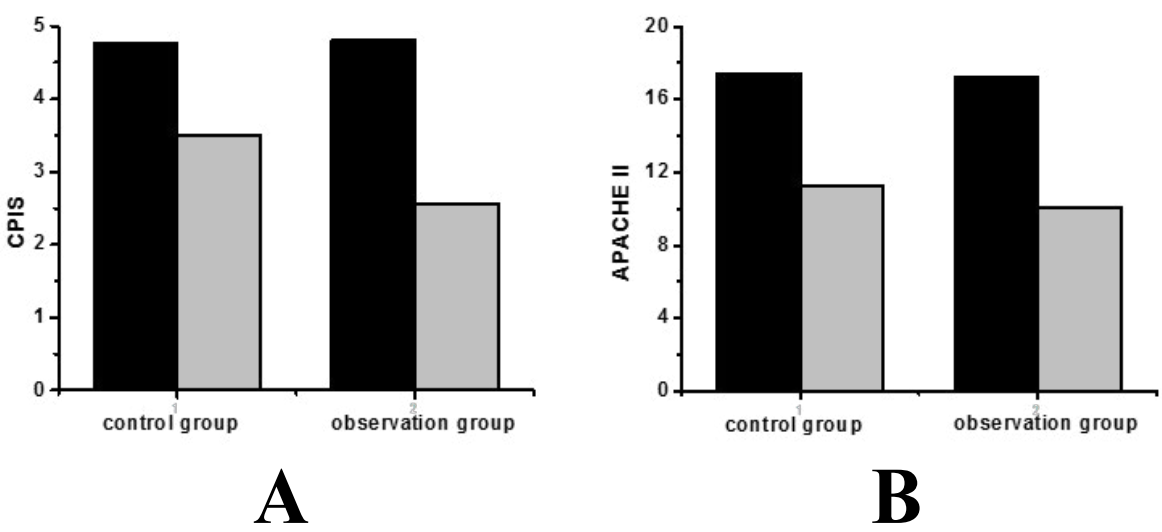

Fig. 2: Comparison of CPIS and APACHE II score between the two groups (A is CPIS and B is APACHE II score)

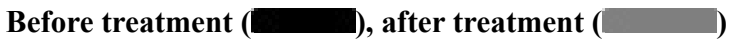

comprehensive rehabilitation treatment (observation group). The success rate of ventilator weaning, the incidence of VAP, the duration of $\mathrm{MV}$, the length of hospital stays in ICU, the CPIS and the APACHE II score were recorded and compared. The results showed that the success rate of ventilator weaning of the observation group was significantly higher than that of the control group. The difference was statistically significant. The incidence of VAP, MV time and the length of hospital stays in ICU were significantly lower in the observation group than in the control group. The difference was statistically significant. Compared with before treatment, the CPIS and APACHE II score of the two groups were significantly lower after treatment, and the difference was statistically significant. The CPIS of the observation group after treatment was significantly lower than that of the control group. The difference was statistically significant, while the APACHE II score of the observation group after treatment was lower than that of the control group, but the difference was not statistically significant.

Therefore, this study found that comprehensive rehabilitation treatment for critical patients is safe and effective and can be used as a routine treatment in clinical ICU, through the study of the impact of comprehensive rehabilitation intervention on the occurrence of VAP in critical patients. However, there are some shortcomings in the research process. For example, the sample data collection is small so the result may have deviation to a certain extent. Therefore, the data capacity will be further increased in the later research process, so that the obtained results can be more valuable.

\section{Acknowledgement:}

None

\section{Conflict of Interests:}

The authors declared no conflict of interest.

\section{REFERENCES}

1. Puerma-Castillo MC, Garcia-Rios MC, Perez-Gomez ME, Aguilar-Ferrandiz ME, Peralta-Ramirez MI. Effectiveness of kinesio taping in addition to conventional rehabilitation treatment on pain, cervical range of motion and quality of life in patients with neck pain: A randomized controlled trial. J Back Musculoskelet Rehabil 2018;31(3):453-64.

2. Vitali I, Genovese O, Conti G, Raffaelli L, D'Addona A, Dell'Anna A, et al. Effectiveness of a ventilator-associated pneumonia prevention bundle in critically ill children. J Biol Regul Homeost Agents 2019;33(2):643-7.

3. Voronina DD, Kulikov AG, Luppova IA, Yarustovskaya OV. The application of general magnetic therapy for the rehabilitation of the patients following the surgical treatment of herniated intervertebral discs. Vopr Kurortol Fizioter Lech Fiz Kult 2017;94(2):24-8.

4. Wheeler PC, Tattersall C. Extracorporeal shockwave therapy plus rehabilitation for patients with chronic plantar fasciitis might reduce pain and improve function but still not lead to increased activity: a case-series study with multiple outcome measures. J Foot Ankle Surg 2018;57(2):339-45.

5. Samalin L, de Chazeron I, Belzeaux R, Llorca PM. Exploratory analysis of the French version of the beliefs about medicines questionnaire in patients with severe mental disorders: Factorial structure and reliability in specific populations of schizophrenic, bipolar and depressive patients. PLoS One 2017;12(3):e0173267.

6. Schmid DA, Allum JH, Sleptsova M, Gross S, Gaab J, WelgeLussen A, et al. Effects of a program of cognitive-behavioural group therapy, vestibular rehabilitation, and psychoeducational explanations on patients with dizziness and no quantified balance deficit, compared to patients with dizziness and a quantified balance deficit. J Psychosom Res 2018;105:21-30.

7. Pol MC, ter Riet G, van Hartingsveldt M, Krose B, de Rooij $\mathrm{SE}$, Buurman BM. Effectiveness of sensor monitoring in an occupational therapy rehabilitation program for older individuals after hip fracture, the SO-HIP trial: study protocol of a three-arm stepped wedge cluster randomized trial. BMC Health Serv Res 2017;17(1):3.

8. Acosta-Olivo C, Siller-Adame A, Tamez-Mata Y, VilchezCavazos F, Pena-Martinez V. Laser Treatment on Acupuncture 
Points Improves Pain and Wrist Functionality in Patients Undergoing Rehabilitation Therapy after Wrist Bone Fracture. A Randomized, Controlled, Blinded Study. Acupunct Electrother Res 2017;42(1):11-25.

9. Lee JH, Kim HJ, Kim YH. Is $\beta$-lactam plus macrolide more effective than $\beta$-lactam plus fluoroquinolone among patients with severe community-acquired pneumonia? A systemic review and meta-analysis. J Korean Med Sci 2017;32(1):7784.

10. Jang JY, Kwon HY, Choi EH, Lee WY, Shim H, Bae KS. Efficacy and toxicity of high-dose nebulized colistin for critically ill surgical patients with ventilator-associated pneumonia caused by multidrug-resistant Acinetobacter baumannii. J Crit Care 2017;40:251-6.
11. Akdogan O, Ersoy Y, Kuzucu C, Gedik E, Togal T, Yetkin F. Assessment of the effectiveness of a ventilator associated pneumonia prevention bundle that contains endotracheal tube with subglottic drainage and cuff pressure monitorization. Braz J Infect Dis 2017;21(3):276-81.

This is an open access article distributed under the terms of the Creative Commons Attribution-NonCommercial-ShareAlike 3.0 License, which allows others to remix, tweak, and build upon the work non-commercially, as long as the author is credited and the new creations are licensed under the identical terms

This article was originally published in a special issue, "Trends in Therapeutic Management of Various Clinical Conditions II" Indian J Pharm Sci 2021:83(2)Spl issue;24-29 